\title{
Acute kidney injury in stable COPD and at exacerbation
}

\author{
This article was published in the following Dove Press journal: \\ International Journal of COPD \\ 28 September 2015 \\ Number of times this article has been viewed
}

\section{MF Barakat ${ }^{\prime}$ \\ HI McDonald' \\ TJ Collier' \\ L Smeeth' \\ D Nitsch' \\ JK Quint ${ }^{1,2}$}

'Faculty of Epidemiology and Population Health, London School of Hygiene and Tropical Medicine, ${ }^{2}$ Department of Respiratory Epidemiology, Occupational Medicine and Public Health, National Heart and Lung Institute, Imperial College London, London, UK
Correspondence: MF Barakat Faculty of Epidemiology and Population Health, London School of Hygiene and Tropical Medicine, Keppel Street, London WCIE 7HT, UK

Email fbarakat86@gmail.com
Background: While acute kidney injury (AKI) alone is associated with increased mortality, the incidence of hospital admission with AKI among stable and exacerbating COPD patients and the effect of concurrent AKI at COPD exacerbation on mortality is not known.

Methods: A total of 189,561 individuals with COPD were identified from the Clinical Practice Research Datalink. Using Poisson and logistic regressions, we explored which factors predicted admission for AKI (identified in Hospital Episode Statistics) in this COPD cohort and concomitant AKI at a hospitalization for COPD exacerbation. Using survival analysis, we investigated the effect of concurrent AKI at exacerbation on mortality $(n=36,107)$ and identified confounding factors.

Results: The incidence of AKI in the total COPD cohort was 128/100,000 person-years. The prevalence of concomitant AKI at exacerbation was $1.9 \%$, and the mortality rate in patients with AKI at exacerbation was 521/1,000 person-years. Male sex, older age, and lower glomerular filtration rate predicted higher risk of AKI or death. There was a 1.80 fold ( $95 \%$ confidence interval: $1.61,2.03)$ increase in adjusted mortality within the first 6 months post COPD exacerbation in patients suffering from AKI and COPD exacerbation compared to those who were AKI free.

Conclusion: In comparison to previous studies on general populations and hospitalizations, the incidence and prevalence of AKI is relatively high in COPD patients. Coexisting AKI at exacerbation is prognostic of poor outcome.

Keywords: acute renal failure, mortality, emphysema, chronic bronchitis, prognosis

\section{Introduction}

COPD affects $9 \%-10 \%$ of people over 40 years of age. ${ }^{1,2}$ It is the fifth biggest killer in the UK, and direct UK health care costs secondary to COPD equate to $£ 805$ million. ${ }^{3}$ High levels of comorbidities, including ischemic heart disease, heart failure (HF), diabetes mellitus (DM), and chronic kidney disease (CKD), contribute to morbidity costs and mortality rates in COPD. ${ }^{4-6} \mathrm{CKD}$ is often seen even in mild COPD cases, and can be largely attributed to both older age and smoking. ${ }^{5,7,8}$ Although CKD is debilitating on its own, its presence in COPD patients is particularly important as many COPD medications are metabolized by the kidney. ${ }^{7}$ Acute kidney injury (AKI) is defined as a rapid decline in renal function from baseline occurring over several hours or days, ${ }^{9}$ more specifically, an absolute rise in the creatinine level by at least $26.4 \mu \mathrm{mol} / \mathrm{L}$ in 48 hours. ${ }^{10}$ People with underlying reduced estimated glomerular filtration rate (eGFR) and even those with mild proteinuria are at increased risk of AKI. ${ }^{11-13}$ Although by definition AKI is reversible, the notion of reversibility is too simplistic; worsening renal function is commonly observed following AKI. ${ }^{9,14}$

Given that CKD is more prevalent in people with COPD than without and given that $\mathrm{CKD}$ is a risk factor for $\mathrm{AKI}$, it is possible that the rate of $\mathrm{AKI}$ is higher among 
COPD patients than in people without COPD. Additionally, at the time of a COPD exacerbation, when gaseous exchange within the lungs may become less effective and carbon dioxide retention can occur, reduced renal blood flow and hence GFR can result. ${ }^{15-18}$ Therefore, it is likely that there is a higher prevalence of AKI in patients hospitalized with COPD exacerbations than general hospitalizations. While AKI has been associated with larger mortality rates in people with community-acquired pneumonia, there are no published studies investigating mortality in COPD patients in the presence of AKI. ${ }^{19}$

Our overarching hypothesis was that people with COPD are at increased risk of AKI. Using linked Clinical Practice Research Datalink (CPRD) and Hospital Episode Statistics (HES) data, we addressed several specific questions: 1) we quantified the incidence and potential risk factors for hospital admission with AKI in a cohort of COPD patients; 2) we determined the prevalence of and possible risk factors for AKI among people hospitalized with a COPD exacerbation; and 3) we investigated mortality rates following hospitalization for COPD exacerbation stratified by AKI at admission, adjusted for confounding factors.

\section{Methods}

\section{Data sources}

CPRD is the world's largest validated computerized database of anonymized longitudinal medical records for primary care. ${ }^{20}$ Data comprise approximately 14 million patients with approximately 5.4 million of them currently alive and registered at 660 primary care practices spread throughout the UK. ${ }^{20}$ Records are derived from the Vision software system and contain complete prescribing and coded diagnostic and clinical information as well as information on tests requested, laboratory results, and referrals made at or following on from each consultation. ${ }^{20}$ The population of patients within CPRD are representative of the UK population with respect to age, sex, and geographical distribution. Over $60 \%$ of the English practices have consented to linkage with HES. HES data contain information on all NHS inpatient admissions in England, with the main and subsidiary reasons for admission coded using the tenth revision of the International Statistical Classification of Diseases and Related Health Problems (ICD-10). ${ }^{21}$

\section{Study population of patients with COPD}

The main study population consisted of individuals over 35 years old, with at least 1 year of historical data, evidence of having ever smoked, and a diagnosis of COPD. COPD was identified in CPRD using validated Read Codes. ${ }^{22}$ Study entry occurred on the latest of the following dates: January 1 , 2004; 1 year after registration with the practice; or 1 year after the practice quality standards were met for inclusion in CPRD. Study exit was the earliest of the following dates: patient transfer-out date, patient death, or December 31, 2012. CPRD records were linked with HES data to identify patients who were hospitalized and the reason for their hospitalization. Patients were excluded if they had no follow-up data or a known history of renal dialysis or transplant (renal replacement therapy) and Read Codes from CPRD of CKD stage 5 or end-stage renal failure (Figure 1).

\section{Identification of first COPD exacerbation during follow-up}

To study the prevalence of and risk factors for concurrent $\mathrm{AKI}$ at the first COPD exacerbation and subsequent mortality, patients within the main study population experiencing at least one COPD exacerbation with available follow-up data were identified (Figure 1). Patients entered this subpopulation at the first record of COPD exacerbation after study entry

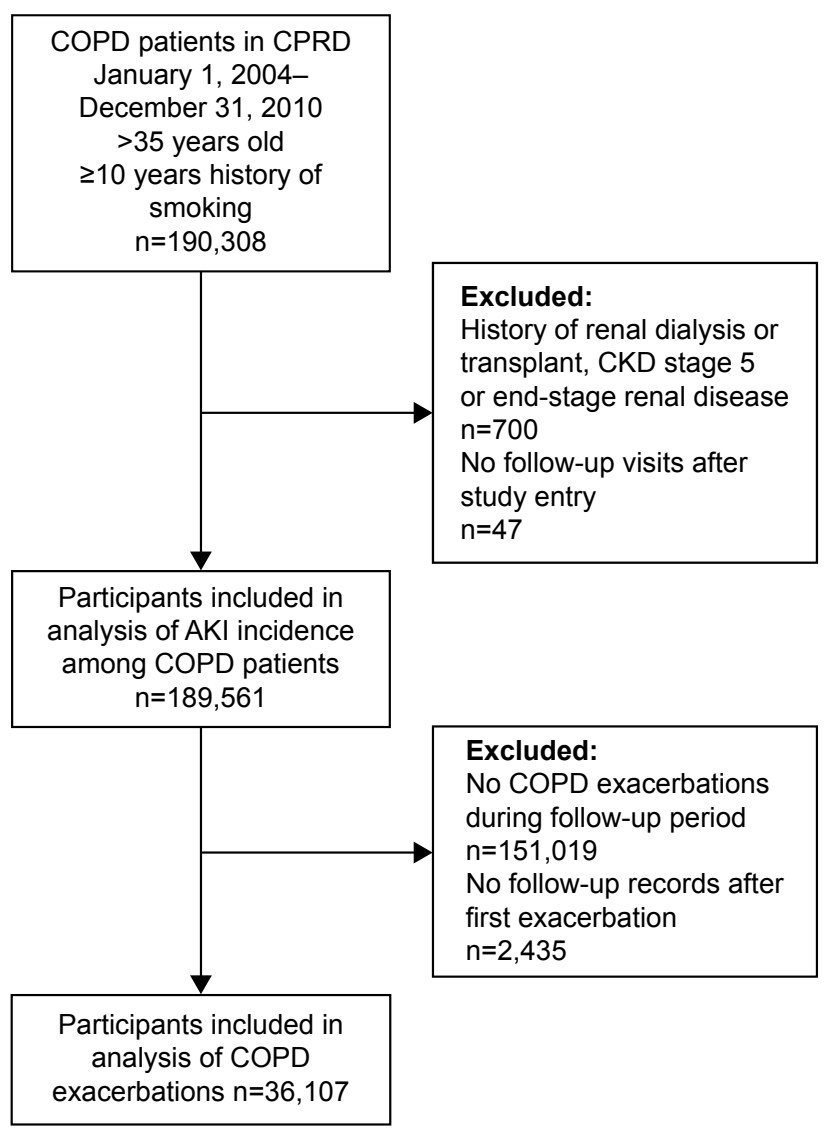

Figure I Flowchart of participant inclusion.

Abbreviations: CPRD, Clinical Practice Research Datalink; CKD, chronic kidney disease; AKI, acute kidney injury; COPD, chronic obstructive pulmonary disease. 
and left at study exit as defined above. COPD exacerbations were identified from HES records as any hospitalization where the primary reason for admission was described using ICD-10 code for infective exacerbation of COPD with ICD-10 code J44 Chronic Obstructive Pulmonary Disease with (Acute) Exacerbation. Repeat COPD exacerbations were not included.

\section{Definition of outcomes}

Similarly, AKI was identified from HES records as any hospitalization where the primary reason for admission was described with ICD-10 code N17 Acute Kidney Failure. For the description of COPD exacerbations, the primary outcome was the presence of coexisting AKI in any diagnostic position in HES records at the time of hospital admission for a COPD exacerbation. For the description of mortality following COPD exacerbation, the outcome was all-cause mortality, identified based on CPRD records.

\section{Explanatory variables}

Demographic data included the patient's age, sex, and region in England. COPD severity was categorized according to Global initiative for chronic Obstructive Lung Disease (GOLD) stage and recorded. ${ }^{23}$ Body mass index (BMI) was categorized as per the World Health Organization classification ${ }^{24}$ : underweight $\left(<18.5 \mathrm{~kg} / \mathrm{m}^{2}\right)$, normal $\left(18.5-24 \mathrm{~kg} / \mathrm{m}^{2}\right)$, overweight (25-29 kg/m²), obese (30-34 kg/m²), moderately obese $\left(35-39 \mathrm{~kg} / \mathrm{m}^{2}\right)$, severely obese $\left(40-44 \mathrm{~kg} / \mathrm{m}^{2}\right)$, and morbidly obese $\left(\geq 45 \mathrm{~kg} / \mathrm{m}^{2}\right)$. Comorbidities were identified by the presence of a diagnostic Read Code in CPRD: hypertension (HTN), DM, angina, previous myocardial infarction, atrial fibrillation, HF, history of coronary artery bypass graft $(\mathrm{CABG})$, and pulmonary HTN. Data regarding COPD and diabetic or cardiovascular medications (antiplatelets, beta blockers, diuretics, angiotensin-converting enzyme inhibitors/ angiotensin receptor blocker, antiarrhythmic, anticoagulant, lipid lowering, nitrate, calcium channel blocker) were identified from primary care prescriptions generated in CPRD.

For the total cohort of patients with COPD, the availability of serum creatinine test results was low, and we used diagnostic Read Codes to identify CKD. The diagnosis and coding of CKD in primary care evolved during the study period. CKD staging based on eGFR was introduced in 2006, but many GPs continued to use the actual renal diagnosis rather than the GFR staging (CKD), thus capturing the renal disease etiology of the patient rather than renal function. We classified patients as having: 1) a diagnosis of CKD stage 3-4 (CKD); 2) a diagnosis of likely renal impairment (renal disease), including microalbuminuria, proteinuria, or previous AKI admissions; or 3) no diagnosis of renal disease (no CKD). For patients with at least one COPD exacerbation, serum creatinine test results were complete, and we estimated eGFR from serum creatinine tests in primary care closest to the date of exacerbation (no less than 2 weeks prior to episode) using the CKD-Epidemiology Collaboration equation. ${ }^{25,26}$

Although smoking is a known risk factor for mortality, CKD, and COPD, we were only able to categorize patients as ex-smokers or current smokers and could not quantify any further than that. As patients had to be ex- or current smokers to be included in the COPD cohort to begin with, we did not use smoking status as an explanatory variable.

\section{Statistical methods}

Poisson regression models were used to estimate associations between potential explanatory variables and crude AKI incidence. Given the large size of the dataset, it is inevitable that there will be a strong evidence of differences across explanatory categories during univariable analysis, and to avoid over adjusting, only age and sex were added to the multivariable model to calculate the observed age and sexadjusted AKI incidence. The association between patient characteristics and prevalence of $\mathrm{AKI}$ at COPD exacerbation was analyzed using logistic regression to calculate crude and adjusted odds ratios. The relationship between AKI and death following COPD exacerbation was investigated using Poisson regression models to calculate rate ratios of death and AKI, crude and adjusted for potential confounders. A forward causal modeling approach was used, with eGFR, age, and sex considered as a priori confounders. Other significant factors such as DM, HF, HTN, myocardial infarction, and CABG all are associated with a risk of CKD. Hence, including them in the multivariable analysis will contribute an element of collinearity. Thus, a simple approach was taken in the multivariable analysis: including only a priori confounders.

Evaluation of missingness was carried out by comparing the observed outcome of interest (AKI) in the group where data on BMI and COPD severity are available to those where they are not. For all models, a complete case analysis approach was used and patients with missing BMI or COPD severity data were excluded. All statistical analyses were performed using Stata 12.1 statistical analysis software (StataCorp LP, College Station, TX, USA).

\section{Ethics}

Ethics approval was obtained from the Independent Scientific Advisory Committee to CPRD (reference 13_086) and the 
London School of Hygiene and Tropical Medicine ethics committee.

\section{Results}

We included 189,561 participants with COPD, of whom 36,107 (19.0\%) had records of at least one COPD exacerbation with follow-up encounters (Figure 1). The mean age of the overall cohort was 64.0 years (SD: 13.6). Most of the cohort (78.9\%) did not have known CKD identified by CPRD Read Codes (Table 1). The cohort was followed up for a median time of 2.3 years.

With regard to missing data, only approximately $4 \%$ BMI data were missing in both the stable and exacerbation of COPD dataset, resulting in an almost negligible effect on the full dataset; therefore, complete case analysis was used to construct any model. A more substantive proportion of $41 \%$ of stable COPD patients were missing COPD severity. There was no evidence of a difference in AKI between individuals with and without COPD severity data. Although missing at random cannot be assumed, we decided to carry out complete case analysis for this cohort. A total of $17.8 \%$ of the exacerbation cohort was missing COPD severity data. There is a clear difference in AKI events between samples with and without this data in the exacerbation cohort; hence, the data are not missing at random. The overall missingness remains below $20 \%$, and for the sake of simplicity, complete case analysis was executed.

\section{Aim I: Incidence of AKI among the cohort of patients with COPD}

Among the total COPD cohort, 1,610 patients experienced hospitalization for AKI, yielding an incidence rate of 128/10,000 person-years (128/105 py, 95\% CI: 121, 134). The incidence of AKI was higher among men than women and rose with increasing age (Table 1).

The observed relationship of COPD severity with AKI incidence demonstrated increasing AKI rates with worsening COPD severity, with a drop in patients with most severe COPD (GOLD stage IV). AKI incidence rates were dramatically higher in patients with CKD $\left(370 / 10^{5}\right.$ py) or renal disease $\left(1,081 / 10^{5} \mathrm{py}\right)$ compared to those with no CKD $\left(41 / 10^{5}\right.$ py).

Comorbidities had an effect on the incidence of AKI in the COPD cohort. The rate of AKI among patients suffering from HF was 6.36 times (95\% CI: 5.76, 7.03) higher than that among patients not diagnosed with HF. Patients with diagnosed CKD or renal disease had a 8.95-fold $(95 \%$ CI: $7.96,10.06)$ or a 26.16 -fold $(95 \%$ CI: $22.48,30.43)$ increase in the rate of AKI compared to those with no CKD, respectively. The increased AKI incidence associated with other comorbidities ranged from a 2.26-fold increase $(95 \%$ CI: $2.02,2.53)$ for angina to a 3.31 -fold increase $(95 \% \mathrm{CI}$ : $2.46,4.46)$ for pulmonary HTN. All studied medications were associated with increased rates of AKI, particularly diuretics and inotropes producing crude risk ratios (RRs) of 7.67 (95\% CI: 6.5, 8.98) and 4.27 (95\% CI: 3.82, 4.79), respectively, with negligible change when adjusted for age and sex.

\section{Aim 2: Prevalence of AKI during hospitalization for exacerbation of COPD}

AKI was present in 704 of 36,107 COPD exacerbations (1.9\%). The odds of AKI in COPD exacerbations were $26 \%$ lower in women compared to men (OR $=0.74,95 \% \mathrm{CI}: 0.63$, 0.86). The prevalence of concurrent AKI increased with age, from $0.4 \%$ among patients aged $<50$ years to $3.5 \%$ among patients $\geq 90$ years of age (crude OR $=9.21,95 \%$ CI: 3.64 , 23.33). Prevalence of AKI was strongly associated with reduced eGFR before and after adjusting for age and sex. AKI was also more prevalent among patients with HTN, DM, atrial fibrillation, and HF in crude analyses. Evidence of association of AKI with HTN and HF remained after adjusting for age, sex, and eGFR (Table 2).

\section{Aim 3:AKI and mortality following COPD exacerbation}

Among the 36,107 patients hospitalized for a COPD exacerbation, 14,447 died during follow-up (40\%). The mean age of those who died was 73.9 years (SD: 9.4). Mortality rates rose with increasing age category from $35 / 1,000$ person-years $\left(35 / 10^{3}\right.$ py) in the youngest to $397 / 10^{3}$ py in the oldest group. Mortality was lower among women than men $(\mathrm{RR}=0.87$, 95\% CI: $0.84,0.90)$, both before and after adjusting for age and eGFR (Table 3).

Reduced eGFR was strongly associated with increased mortality, but the relationship appeared J-shaped, with higher mortality among patients with eGFR $\geq 90$ than patients with eGFR $60-89$ or $30-59 \mathrm{~mL} / \mathrm{min} / 1.73 \mathrm{~m}^{2}$ after adjusting for age and sex. BMI was also strongly associated with mortality, with the highest mortality found in underweight individuals in comparison to those with normal BMI (adjusted RR $=1.41,95 \% \mathrm{CI}: 1.30,1.52$ ). There was a graded association between worsening severity of COPD and higher mortality. Diagnosed HTN or DM, a history of CABG, and prescriptions of COPD medication, beta blockers, vasodilators, nitrates, antiarrhythmics, and lipid-lowering 
Table I Baseline characteristics of all patients with COPD $(n=\mid 89,56 I)$ and incidence AKI $(n=|, 6| 0)$

\begin{tabular}{|c|c|c|c|c|c|c|}
\hline $\begin{array}{l}\text { Baseline patient } \\
\text { characteristics }\end{array}$ & $\begin{array}{l}\text { Total at } \\
\text { baseline, n (\%) }\end{array}$ & $\begin{array}{l}\text { With AKI, n } \\
\text { (rate/ I00,000 py) }\end{array}$ & $\begin{array}{l}\text { Crude rate ratio } \\
\text { of AKI }(95 \% \mathrm{Cl})\end{array}$ & $P$-value ${ }^{a}$ & $\begin{array}{l}\text { Adjusted }^{\mathrm{b}} \text { rate ratio } \\
\text { of } \mathrm{AKI}(95 \% \mathrm{Cl})\end{array}$ & $P$-value ${ }^{a}$ \\
\hline \multicolumn{7}{|l|}{ Sex } \\
\hline Male & $90,466(47.7)$ & $926(157)$ & I (reference) & \multirow[t]{2}{*}{$<0.001$} & I (reference) & \multirow[t]{2}{*}{$<0.001$} \\
\hline Female & 99,095 (52.3) & $684(95)$ & $0.65(0.59,0.72)$ & & $0.66(0.60,0.73)$ & \\
\hline \multicolumn{7}{|l|}{ Age (years) } \\
\hline$<50$ & 30,761 (16.2) & $30(14)$ & I (reference) & \multirow[t]{6}{*}{$<0.001$} & I (reference) & \multirow[t]{6}{*}{$<0.00$ I } \\
\hline $50-59$ & 37,538 (19.8) & $125(38)$ & $3.27(2.20,4.88)$ & & $3.19(2.14,4.75)$ & \\
\hline $60-69$ & $49,422(26.1)$ & $354(102)$ & $7.43(5.12,10.79)$ & & $7.14(4.92,10.37)$ & \\
\hline $70-79$ & $46,174(24.4)$ & $640(219)$ & $15.84(10.98,22.84)$ & & $15.30(10.61,22.06)$ & \\
\hline $80-89$ & $22,822(12.0)$ & $423(368)$ & $26.68(18.43,38.64)$ & & $26.42(18.25,38.27)$ & \\
\hline$\geq 90$ & $2,844(1.5)$ & $38(380)$ & $27.52(17.04,44.4 I)$ & & $28.66(17.76,46.27)$ & \\
\hline \multicolumn{7}{|l|}{ Region } \\
\hline London & $20,819(11.0)$ & $199(146)$ & I (reference) & \multirow[t]{4}{*}{$<0.001$} & I (reference) & \multirow[t]{4}{*}{$<0.001$} \\
\hline Midlands & 31,903 (16.8) & $236(114)$ & $0.78(0.64,0.94)$ & & $0.77(0.64,0.93)$ & \\
\hline North England & $51,093(27.0)$ & 487 (I42) & $0.97(0.83,1.15)$ & & $1.02(0.86,1.20)$ & \\
\hline South England & $85,746(45.2)$ & $688(120)$ & $0.82(0.70,0.96)$ & & $0.80(0.68,0.93)$ & \\
\hline \multicolumn{7}{|l|}{ Comorbidities } \\
\hline \multicolumn{7}{|l|}{ Body mass index } \\
\hline Underweight & $5,3 \mid 3(2.8)$ & $33(106)$ & $1.29(0.91,1.85)$ & \multirow[t]{7}{*}{$<0.001$} & I.42 $(0.99,2.02)$ & \multirow[t]{7}{*}{$<0.001$} \\
\hline Normal & 76,011 (40.I) & $418(82)$ & I (reference) & & I (reference) & \\
\hline Overweight & $64,683(34.1)$ & $592(134)$ & $1.63(1.44,1.85)$ & & $1.43(1.26,1.63)$ & \\
\hline Moderately obese & $24,846(13.1)$ & $299(178)$ & $2.16(I .87,2.5 I)$ & & $2.15(1.85,2.49)$ & \\
\hline Severely obese & $7,324(3.9)$ & $123(25 \mid)$ & $3.06(2.50,3.74)$ & & $3.80(3.10,4.65)$ & \\
\hline Morbidly obese & 3,149 (I.7) & $55(264)$ & $3.22(2.43,4.26)$ & & $5.17(3.89,6.86)$ & \\
\hline Missing & $8,138(4.3)$ & $90(238)$ & - & & - & \\
\hline \multicolumn{7}{|c|}{ COPD severity (GOLD stage) } \\
\hline I & $24,507(12.9)$ & $145(8 \mid)$ & I (reference) & \multirow[t]{5}{*}{$<0.001$} & I (reference) & \multirow[t]{5}{*}{$<0.001$} \\
\hline ॥ & $53,697(28.3)$ & $514(138)$ & $1.70(1.41,2.04)$ & & $\mathrm{I} .42(\mathrm{I} .18, \mathrm{I} .7 \mathrm{I})$ & \\
\hline III & $26,626(14.1)$ & $295(172)$ & $2.12(1.74,2.59)$ & & $1.55(1.27,1.90)$ & \\
\hline IV & $7,007(3.7)$ & $51(126)$ & $1.56(1.13,2.14)$ & & $1.15(0.84,1.59)$ & \\
\hline Missing & $77,724(41.0)$ & $605(123)$ & & & & \\
\hline Hypertension & $89,824(47.4)$ & $1,161(191)$ & $2.75(2.47,3.07)$ & $<0.001$ & $1.89(1.69,2.11)$ & $<0.001$ \\
\hline Diabetes mellitus & $33,042(17.4)$ & $613(272)$ & $2.82(2.55,3.12)$ & $<0.001$ & $2.50(2.26,2.77)$ & $<0.001$ \\
\hline Angina & $25,291(13.3)$ & $398(226)$ & $2.26(2.02,2.53)$ & $<0.001$ & $1.48(1.32,1.66)$ & $<0.001$ \\
\hline Myocardial infarction & $16,872(8.9)$ & $333(288)$ & $2.90(2.57,3.27)$ & $<0.001$ & $1.92(1.70,2.17)$ & $<0.001$ \\
\hline Atrial fibrillation & $22,065(11.6)$ & $457(330)$ & $3.20(2.87,3.56)$ & $<0.001$ & $1.84(1.64,2.05)$ & $<0.001$ \\
\hline Heart failure & $21,778(11.5)$ & $652(537)$ & $6.36(5.76,7.03)$ & $<0.001$ & $3.82(3.44,4.24)$ & $<0.001$ \\
\hline Coronary artery bypass graft & $5,305(2.8)$ & $130(366)$ & $3.03(2.53,3.64)$ & $<0.001$ & $2.13(1.78,2.56)$ & $<0.001$ \\
\hline Pulmonary hypertension & $1,963(1.0)$ & $45(4 \mid 6)$ & $3.31(2.46,4.46)$ & $<0.001$ & $2.37(1.76,3.18)$ & $<0.001$ \\
\hline \multicolumn{7}{|l|}{ Kidney disease } \\
\hline CKD stage $3-4$ & $35,090(18.5)$ & $918(370)$ & $8.95(7.96,10.06)$ & $<0.001$ & $5.90(5.22,6.67)$ & $<0.001$ \\
\hline Renal disease $^{c}$ & $4,835(2.6)$ & $286(I, 08 I)$ & $26.16(22.48,30.43)$ & & $19.67(16.87,22.92)$ & \\
\hline Medications & & & & & & \\
\hline COPD medications & $160,12 \mid(84.5)$ & $\mathrm{I}, 428(135)$ & $1.51(1.29,1.76)$ & $<0.001$ & $1.38(1.18,1.61)$ & 0.001 \\
\hline Antiplatelets & $|05,83|(55.8)$ & $\mathrm{I}, 184(215)$ & $3.59(3.21,4.01)$ & $<0.001$ & $2.08(1.86,2.34)$ & $<0.001$ \\
\hline Antidiabetes medications & $23,591(12.5)$ & $495(3 I I)$ & $3.06(2.75,3.40)$ & $<0.001$ & $2.85(2.56,3.17)$ & $<0.001$ \\
\hline Beta blockers & $61,155(32.3)$ & $787(184)$ & $1.85(1.68,2.04)$ & $<0.001$ & $1.53(1.39,1.69)$ & $<0.001$ \\
\hline Diuretics & $101,116(53.3)$ & $\mathrm{I}, 437(220)$ & $7.67(6.55,8.98)$ & $<0.001$ & $4.72(4.00,5.56)$ & $<0.001$ \\
\hline ACEi/ARB & $79,359(41.9)$ & $\mathrm{I}, 155(217)$ & $3.46(3.10,3.86)$ & $<0.001$ & $2.37(2.12,2.65)$ & $<0.001$ \\
\hline Vasodilators & $11,292(6.0)$ & $43(53)$ & $0.40(0.29,0.54)$ & $<0.001$ & $0.53(0.39,0.7 I)$ & $<0.001$ \\
\hline Alpha blockers & $7,338(3.9)$ & $167(342)$ & $2.87(2.44,3.36)$ & $<0.00$ I & $2.00(I .7 I, 2.35)$ & $<0.001$ \\
\hline Inotropes & $15,477(8.2)$ & $398(444)$ & $4.27(3.82,4.79)$ & $<0.001$ & $2.40(2.14,2.70)$ & $<0.001$ \\
\hline Nitrates & $26,114(13.8)$ & $395(236)$ & $2.12(1.89,2.38)$ & $<0.001$ & $1.59(1.4 \mathrm{I}, \mathrm{I} .78)$ & 0.002 \\
\hline Calcium channel blockers & $58,611(30.9)$ & 745 (I84) & $1.81(1.64,2.00)$ & $<0.001$ & $1.36(1.24,1.5 \mathrm{I})$ & 0.001 \\
\hline Antiarrhythmic & $7,633(4.0)$ & $182(384)$ & $3.25(2.79,3.80)$ & $<0.001$ & $2.18(1.87,2.55)$ & $<0.001$ \\
\hline Anticoagulants & $21,977(11.6)$ & $454(3 \mid 8)$ & $3.07(2.75,3.42)$ & $<0.001$ & $2.05(1.84,2.29)$ & $<0.001$ \\
\hline Lipid lowering & $87,747(46.2)$ & $\mathrm{I}, 067(175)$ & $2.09(1.88,2.32)$ & $<0.001$ & $1.64(1.47,1.82)$ & $<0.001$ \\
\hline
\end{tabular}

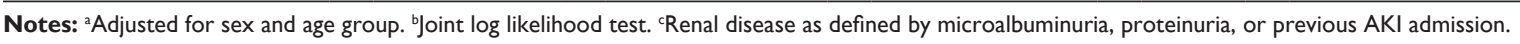

Abbreviations: COPD, chronic obstructive pulmonary disease; AKI, acute kidney injury; py, person-years; $\mathrm{Cl}$, confidence interval; GOLD, Global initiative for chronic Obstructive Lung Disease; CKD, chronic kidney disease; ACEi, angiotensin-converting enzyme inhibitors; ARB, angiotensin II receptor blocker. 
Table 2 Baseline characteristics of patients with COPD exacerbation $(n=36,107)$ and odds ratios for coexistence of AKI ( $n=704)$

\begin{tabular}{|c|c|c|c|c|c|c|}
\hline $\begin{array}{l}\text { Baseline patient } \\
\text { characteristics }\end{array}$ & $\begin{array}{l}\text { Total at } \\
\text { baseline, n (\%) }\end{array}$ & $\begin{array}{l}\text { Concurrent } \\
\text { AKI, n (\%) }\end{array}$ & $\begin{array}{l}\text { Crude odds ratio } \\
(95 \% \mathrm{Cl})\end{array}$ & $P$-value ${ }^{a}$ & $\begin{array}{l}\text { Adjusted }^{\mathrm{b}} \text { odds } \\
\text { ratio }(95 \% \mathrm{Cl})\end{array}$ & $P$-value \\
\hline \multicolumn{7}{|l|}{ Sex } \\
\hline Male & $19,235(53.3)$ & $427(2.2)$ & I (reference) & \multirow[t]{2}{*}{$<0.001$} & I (reference) & \multirow[t]{2}{*}{$<0.001$} \\
\hline Female & $16,872(46.7)$ & $277(1.6)$ & $0.74(0.63,0.86)$ & & $0.68(0.58,0.80)$ & \\
\hline \multicolumn{7}{|l|}{ Age (years) } \\
\hline$<50$ & $\mathrm{I}, 547(4.3)$ & $6(0.4)$ & I (reference) & \multirow[t]{6}{*}{$<0.00$ I } & I (reference) & \multirow[t]{6}{*}{0.066} \\
\hline $50-59$ & 4,989 (I3.8) & $37(0.7)$ & $1.92(0.8 \mathrm{I}, 4.56)$ & & I.55 $(0.65,3.7 \mathrm{I})$ & \\
\hline $60-69$ & $10,404(28.8)$ & $138(1.3)$ & $3.45(1.52,7.83)$ & & $1.93(0.84,4.45)$ & \\
\hline $70-79$ & $12,496(34.6)$ & $303(2.4)$ & $6.38(2.84,14.3)$ & & $2.28(0.99,5.25)$ & \\
\hline $80-89$ & $6,151(17.0)$ & $202(3.3)$ & $8.72(3.86,19.68)$ & & $2.37(1.02,5.50)$ & \\
\hline$\geq 90$ & $520(1.4)$ & $18(3.5)$ & $9.21(3.64,23.33)$ & & I.88 $(0.72,4.93)$ & \\
\hline \multicolumn{7}{|l|}{ Region } \\
\hline London & $3,81 I(10.6)$ & $88(2.3)$ & I (reference) & \multirow[t]{4}{*}{0.005} & I (reference) & \multirow[t]{4}{*}{0.035} \\
\hline Midlands & $5,453(15.1)$ & $109(2.0)$ & $0.86(0.64,1.15)$ & & $0.84(0.63,1.13)$ & \\
\hline North England & $12,054(33.4)$ & $193(1.6)$ & $0.69(0.53,0.89)$ & & $0.89(0.70,1.14)$ & \\
\hline South England & $14,789(4 \mid .0)$ & $314(2.1)$ & $0.92(0.72,1.17)$ & & $0.7 \mathrm{I}(0.55,0.92)$ & \\
\hline \multicolumn{7}{|l|}{ Comorbidities } \\
\hline \multicolumn{7}{|l|}{ Body mass index } \\
\hline Underweight & I,4I (3.9) & $22(1.6)$ & $0.94(0.60,1.46)$ & \multirow[t]{7}{*}{$<0.00 \mathrm{I}$} & $1.18(0.75,1.86)$ & \multirow[t]{7}{*}{0.002} \\
\hline Normal & $14,296(39.6)$ & $236(1.7)$ & I (reference) & & I (reference) & \\
\hline Overweight & $12,056(33.4)$ & $221(1.8)$ & I.I I $(0.92$, I.34) & & $0.91(0.76,1.11)$ & \\
\hline Moderately obese & $4,893(13.6)$ & $117(2.4)$ & $1.46(1.17,1.83)$ & & $1.23(0.98,1.55)$ & \\
\hline Severely obese & $1,345(3.7)$ & $38(2.8)$ & $1.73(1.22,2.45)$ & & $1.51(1.05,2.17)$ & \\
\hline Morbidly obese & $643(1.8)$ & $20(3.1)$ & $1.91(1.20,3.04)$ & & $2.13(1.31,3.46)$ & \\
\hline Missing & $\mathrm{I}, 456(4.0)$ & $50(3.4)$ & - & & - & \\
\hline \multicolumn{7}{|l|}{ COPD severity (GOLD stage) } \\
\hline I & $3,669(10.2)$ & $50(1.4)$ & I (reference) & \multirow[t]{5}{*}{0.239} & I (reference) & \multirow[t]{5}{*}{0.292} \\
\hline$\|$ & $13,690(37.9)$ & $253(1.9)$ & $1.36(1.00,1.85)$ & & $1.34(0.98,1.83)$ & \\
\hline III & $9,473(26.2)$ & $165(1.7)$ & $1.28(0.93,1.76)$ & & $1.26(0.91,1.75)$ & \\
\hline IV & $2,844(7.9)$ & $50(1.8)$ & $1.30(0.87,1.92)$ & & $1.37(0.91,2.07)$ & \\
\hline Missing & $6,431(17.8)$ & $186(2.9)$ & - & & - & \\
\hline Hypertension & $19,517(54.1)$ & $480(2.5)$ & $1.84(1.57,2.16)$ & $<0.001$ & $1.35(1.14,1.59)$ & $<0.001$ \\
\hline Diabetes mellitus & $8,044(22.3)$ & $193(2.4)$ & $1.32(1.12,1.57)$ & 0.001 & $1.17(0.98,1.39)$ & 0.083 \\
\hline Angina & $7,252(20.1)$ & $137(1.9)$ & $0.96(0.80,1.16)$ & 0.676 & $0.74(0.61,0.89)$ & 0.002 \\
\hline Myocardial infarction & $5,382(14.9)$ & $118(2.2)$ & $1.15(0.94, I .41)$ & 0.163 & $0.85(0.69,1.05)$ & 0.125 \\
\hline Atrial fibrillation & $7,028(19.5)$ & $186(2.7)$ & $1.50(1.27,1.78)$ & $<0.00 \mathrm{I}$ & $1.08(0.91,1.29)$ & 0.382 \\
\hline Heart failure & $7,645(21.2)$ & $258(3.4)$ & $2.19(1.88,2.56)$ & $<0.001$ & $1.27(1.08,1.50)$ & 0.004 \\
\hline Coronary artery bypass graft & $\mathrm{I}, 557(4.3)$ & $32(2.1)$ & $1.06(0.74, I .5 \mathrm{I})$ & 0.758 & $0.82(0.57,1.19)$ & 0.304 \\
\hline Pulmonary hypertension & $942(2.6)$ & $23(2.4)$ & $1.27(0.83,1.93)$ & 0.270 & $1.13(0.73,1.73)$ & 0.591 \\
\hline CKD $\left(\mathrm{eGFR} \mathrm{mL} / \mathrm{kg} / \mathrm{l} .73 \mathrm{~m}^{2}\right)$ & & & & & & \\
\hline$\geq 90$ & $6,320(17.5)$ & $35(0.6)$ & I (reference) & $<0.00 \mathrm{I}$ & I (reference) & $<0.00 \mathrm{I}$ \\
\hline $60-89$ & $16,885(46.8)$ & $145(0.9)$ & $1.56(1.07,2.25)$ & & $1.31(0.89,1.93)$ & \\
\hline $30-59$ & $|I, 48|(3 \mid .8)$ & $34 I(3.0)$ & $5.50(3.88,7.79)$ & & $4.35(2.96,6.38)$ & \\
\hline $15-29$ & $\mathrm{I}, 258(3.5)$ & $128(10.2)$ & $20.30(13.92,29.72)$ & & $15.80(10.40,24.01)$ & \\
\hline$<15$ & $163(0.5)$ & $55(33.7)$ & $91.45(57.47,145.52)$ & & $73.74(44.97,120.93)$ & \\
\hline Medications & & & & & & \\
\hline COPD medications & $35,483(98.3)$ & $679(1.9)$ & $0.47(0.31,0.70)$ & $<0.001$ & $0.54(0.36,0.83)$ & 0.005 \\
\hline Antiplatelets & $22,338(61.9)$ & $480(2.2)$ & $1.33(1.13,1.56)$ & 0.001 & $0.94(0.79,1.11)$ & 0.433 \\
\hline Antidiabetes medications & $5,683(15.7)$ & $153(2.7)$ & $1.50(1.25,1.80)$ & $<0.00 \mathrm{I}$ & $1.32(1.09,1.59)$ & 0.004 \\
\hline Beta blockers & II,746 (32.5) & $274(2.3)$ & $1.33(1.14,1.55)$ & $<0.001$ & $1.09(0.93,1.27)$ & 0.304 \\
\hline Diuretics & $25,82 ।(7 \mid .5)$ & $616(2.4)$ & $2.83(2.26,3.55)$ & $<0.001$ & $1.59(1.25,2.01)$ & $<0.001$ \\
\hline ACEi/ARB & $19,347(53.6)$ & $468(2.4)$ & $1.74(1.48,2.03)$ & $<0.001$ & $1.23(1.05,1.45)$ & 0.012 \\
\hline Vasodilators & I,730 (4.8) & $14(0.8)$ & $0.40(0.23,0.68)$ & 0.001 & $0.53(0.31,0.91)$ & 0.021 \\
\hline Alpha blockers & I,768 (4.9) & $59(3.3)$ & $1.80(1.38,2.36)$ & $<0.001$ & $1.20(0.90,1.59)$ & 0.218 \\
\hline Inotropes & $5,417(15.0)$ & $161(3.0)$ & $1.70(1.42,2.03)$ & $<0.001$ & $1.19(0.99,1.43)$ & 0.068 \\
\hline Nitrates & $7,115(19.7)$ & $129(1.8)$ & $0.78(0.64,0.96)$ & 0.021 & $0.77(0.62,0.95)$ & 0.016 \\
\hline Calcium channel blockers & $13,405(37.1)$ & $312(2.3)$ & $1.29(1.05,1.57)$ & 0.013 & $1.31(1.07,1.61)$ & 0.010 \\
\hline Antiarrhythmic & $2,625(7.3)$ & $60(2.3)$ & $1.19(0.91,1.56)$ & 0.197 & $0.85(0.64, I .11)$ & 0.230 \\
\hline Anticoagulants & $6,976(19.3)$ & $168(2.4)$ & $1.32(1.10,1.57)$ & 0.002 & $1.03(0.86,1.23)$ & 0.768 \\
\hline Lipid lowering & $20,962(58.1)$ & 405 (1.9) & $0.98(0.84,1.14)$ & 0.775 & $1.01(0.86,1.17)^{* *}$ & 0.941 \\
\hline
\end{tabular}

Notes: ajoint log likelihood test. ${ }^{b}$ Adjusted for sex, age group, and eGFR. **Evidence of collinearity with eGFR, adjusted for age and sex only.

Abbreviations: COPD, chronic obstructive pulmonary disease; AKI, acute kidney injury; $\mathrm{Cl}$, confidence interval; GOLD, Global initiative for chronic Obstructive Lung Disease; CKD, chronic kidney disease; eGFR, estimated glomerular filtration rate; ACEi, angiotensin-converting enzyme inhibitors; ARB, angiotensin receptor blocker. 
Table 3 Univariable and multivariable Poisson regression analyses of the association of baseline characteristics with mortality following COPD exacerbation $(n=36,107)$

\begin{tabular}{|c|c|c|c|c|c|}
\hline $\begin{array}{l}\text { Baseline patient } \\
\text { characteristics }\end{array}$ & $\begin{array}{l}\text { Deaths, } n \text { (rate/I,000 } \\
\text { person-years) }\end{array}$ & $\begin{array}{l}\text { Crude rate ratio } \\
(95 \% \mathrm{Cl})\end{array}$ & $P$-value ${ }^{a}$ & $\begin{array}{l}\text { Adjusted }{ }^{\mathrm{b}} \text { rate } \\
\text { ratio }(95 \% \mathrm{Cl})\end{array}$ & $P$-value ${ }^{b}$ \\
\hline \multicolumn{6}{|l|}{ Sex } \\
\hline Male & $8,102(151)$ & I (reference) & \multirow[t]{2}{*}{$<0.001$} & I (reference) & \multirow[t]{2}{*}{$<0.001$} \\
\hline Female & $6,345(131)$ & $0.87(0.84,0.90)$ & & $0.87(0.84,0.90)$ & \\
\hline \multicolumn{6}{|l|}{ Age (years) } \\
\hline$<50$ & $155(35)$ & I (reference) & \multirow[t]{6}{*}{$<0.001$} & I (reference) & \multirow[t]{6}{*}{$<0.001$} \\
\hline $50-59$ & $910(58)$ & $1.67(1.41,1.98)$ & & $1.73(1.46,2.05)$ & \\
\hline $60-69$ & $3,291(103)$ & $2.97(2.53,3.49)$ & & $3.21(2.73,3.77)$ & \\
\hline $70-79$ & $5,831(166)$ & $4.79(4.08,5.62)$ & & $5.24(4.45,6.17)$ & \\
\hline $80-89$ & $3,864(274)$ & $7.88(6.71,9.25)$ & & $8.37(7.10,9.88)$ & \\
\hline$\geq 90$ & $396(397)$ & II .44 (9.50, I 3.78) & & $11.69(9.66,14.14)$ & \\
\hline \multicolumn{6}{|l|}{ Region } \\
\hline London & $1,413(132)$ & I (reference) & \multirow[t]{4}{*}{$<0.001$} & I (reference) & \multirow[t]{4}{*}{$<0.001$} \\
\hline Midlands & $2,164(146)$ & $1.11(1.04,1.19)$ & & $1.13(1.06,1.21)$ & \\
\hline North England & $5,899(144)$ & $1.09(1.03,1.16)$ & & $1.09(1.03,1.15)$ & \\
\hline South England & 4,97I (I39) & $1.05(0.99,1.12)$ & & $1.13(1.07,1.20)$ & \\
\hline Acute kidney injury & $472(5 \mid 2)$ & 3.7I $(3.39,4.07)$ & $<0.001$ & $2.47(2.24,3.73)$ & $<0.001$ \\
\hline \multicolumn{6}{|l|}{ Body mass index } \\
\hline Underweight & $75 I(201)$ & $1.37(1.27,1.48)$ & \multirow[t]{7}{*}{$<0.001$} & $1.41(1.30,1.52)$ & \multirow[t]{7}{*}{$<0.001$} \\
\hline Normal & $5,848(147)$ & I (reference) & & I (reference) & \\
\hline Overweight & $4,425(127)$ & $0.86(0.83,0.90)$ & & $0.82(0.79,0.85)$ & \\
\hline Moderately obese & I,726 (II8) & $0.80(0.76,0.85)$ & & $0.83(0.78,0.87)$ & \\
\hline Severely obese & $478(116)$ & $0.79(0.72,0.87)$ & & $0.88(0.80,0.96)$ & \\
\hline Morbidly obese & $229(112)$ & $0.76(0.67,0.87)$ & & $0.99(0.87,1.13)$ & \\
\hline Missing & $990(34 I)$ & - & & - & \\
\hline \multicolumn{6}{|l|}{ COPD severity (GOLD stage) } \\
\hline I & $886(82)$ & I (reference) & \multirow[t]{5}{*}{$<0.001$} & I (reference) & \multirow[t]{5}{*}{$<0.001$} \\
\hline ॥ & $4,095(99)$ & $1.21(1.13,1.30)$ & & $1.18(1.10,1.27)$ & \\
\hline III & $3,983(140)$ & $1.72(2.14,2.52)$ & & $1.63(1.52,1.75)$ & \\
\hline IV & $1,580(189)$ & $2.32(2.14,2.52)$ & & $2.30(2.11,2.50)$ & \\
\hline Missing & $3,903(295)$ & - & & - & \\
\hline Hypertension & $7,654(136)$ & $0.93(0.90,0.96)$ & $<0.00$ I & $0.82(0.79,0.84)$ & $<0.001$ \\
\hline Diabetes mellitus & $3,058(122)$ & $0.83(0.79,0.86)$ & $<0.00 \mathrm{I}$ & $0.84(0.8 \mathrm{I}, 0.87)$ & $<0.001$ \\
\hline Angina & $3,199(146)$ & $1.04(1.00,1.09)$ & 0.030 & $0.93(0.90,0.97)$ & $<0.00$ I \\
\hline Myocardial infarction & $2,613(168)$ & $1.23(1.18,1.28)$ & $<0.00$ I & $1.08(1.03,1.12)$ & $<0.001$ \\
\hline Atrial fibrillation & $3,46 I(|7|)$ & $1.27(1.22,1.32)$ & $<0.001$ & $1.00(0.96,1.04)$ & 0.844 \\
\hline Heart failure & $4,394(2 / 2)$ & $1.72(1.66,1.78)$ & $<0.00$ I & $1.37(1.32,1.42)$ & $<0.001$ \\
\hline Coronary artery bypass graft & $605(127)$ & $0.89(0.82,0.97)$ & 0.007 & $0.85(0.78,0.92)$ & $<0.001$ \\
\hline Pulmonary hypertension & $567(207)$ & $1.49(1.37,1.62)$ & $<0.001$ & $1.46(1.34,1.59)$ & $<0.001$ \\
\hline \multicolumn{6}{|l|}{ CKD (eGFR in $\left.\mathrm{mL} / \mathrm{kg} / \mathrm{l} .73 \mathrm{~m}^{2}\right)$} \\
\hline$\geq 90$ & I,74I (I03) & I (reference) & \multirow[t]{5}{*}{$<0.001$} & I (reference) & \multirow[t]{5}{*}{$<0.001$} \\
\hline$\geq 60$ and $<90$ & $5,981(114)$ & $1.14(1.08,1.20)$ & & $0.70(0.66,0.74)$ & \\
\hline$\geq 30$ and $<60$ & $5,763(\mid 8 I)$ & $1.76(1.67,1.86)$ & & $0.81(0.76,0.86)$ & \\
\hline$\geq 15$ and $<30$ & $842(360)$ & $3.50(3.23,3.80)$ & & $1.42(1.30,1.55)$ & \\
\hline$<15$ & $120(476)$ & $4.63(3.85,5.57)$ & & $1.84(1.67,2.44)$ & \\
\hline COPD medications & $|4|||$, (I39) & $0.45(0.40,0.50)$ & $<0.00$ I & $0.54(0.49,0.60)$ & $<0.001$ \\
\hline Antiplatelets & $9,421(145)$ & $1.08(1.04,1.11)$ & $<0.00 \mathrm{I}$ & $0.87(0.84,0.90)$ & $<0.001$ \\
\hline Antidiabetes medications & $2,211(125)$ & $0.87(0.83,0.91)$ & $<0.00 \mathrm{I}$ & $0.92(0.88,0.96)$ & $<0.001$ \\
\hline Beta blockers & $4,351(132)$ & $0.91(0.88,0.94)$ & $<0.00$ I & $0.89(0.86,0.92)$ & $<0.001$ \\
\hline Diuretics & $11,744(161)$ & $1.73(1.66,1.80)$ & $<0.001$ & $1.29(1.24,1.35)$ & $<0.001$ \\
\hline ACEi/ARB & $7,959(|4|)$ & $1.00(0.97,1.03)$ & 0.876 & $0.87(0.84,0.90)$ & $<0.001$ \\
\hline Vasodilators & $439(83)$ & $0.58(0.52,0.63)$ & $<0.00$ I & $0.75(0.68,0.82)$ & $<0.001$ \\
\hline Alpha blockers & $773(160)$ & $1.14(1.06,1.22)$ & 0.001 & $0.99(0.92,1.06)$ & 0.730 \\
\hline Inotropes & $3,036(203)$ & $1.55(1.49,1.61)$ & $<0.00 \mathrm{I}$ & $1.22(1.17,1.27)$ & $<0.001$ \\
\hline Nitrates & $3,095(148)$ & $1.06(1.12,1.11)$ & 0.002 & $1.00(0.96,1.04)$ & 0.961 \\
\hline
\end{tabular}


Table 3 (Continued)

\begin{tabular}{|c|c|c|c|c|c|}
\hline $\begin{array}{l}\text { Baseline patient } \\
\text { characteristics }\end{array}$ & $\begin{array}{l}\text { Deaths, } \mathrm{n} \text { (rate/ I,000 } \\
\text { person-years) }\end{array}$ & $\begin{array}{l}\text { Crude rate ratio } \\
(95 \% \mathrm{Cl})\end{array}$ & $P$-value ${ }^{a}$ & $\begin{array}{l}\text { Adjusted }^{\mathrm{b}} \text { rate } \\
\text { ratio }(95 \% \mathrm{Cl})\end{array}$ & $P$-value ${ }^{b}$ \\
\hline Calcium channel blockers & $5,091(130)$ & $0.87(0.85,0.91)$ & $<0.00$ I & $0.83(0.80,0.86)$ & $<0.001$ \\
\hline Antiarrhythmic & I,30I (139) & $0.66(0.64,0.68)$ & $<0.00$ I & $0.68(0.65,0.70)$ & $<0.001$ \\
\hline Anticoagulants & $3,156(153)$ & $1.06(\mathrm{I} .02, \mathrm{I} . \mathrm{II})$ & 0.002 & $1.13(1.08,1.18)$ & $<0.001$ \\
\hline Lipid lowering & $7,534(118)$ & $0.87(0.84,0.91)$ & $<0.001$ & $0.91(0.87,0.95)$ & $<0.001$ \\
\hline \multicolumn{6}{|l|}{ Time from exacerbation } \\
\hline$<6$ months & & I (reference) & $<0.001$ & I (reference) & $<0.001$ \\
\hline$\geq 6$ months & & $0.020(0.19,0.0 .21)$ & & $0.024(0.023,0.025)$ & \\
\hline
\end{tabular}

Notes: ajoint log likelihood test. ${ }^{b}$ Adjusted for sex, age group, and eGFR.

Abbreviations: COPD, chronic obstructive pulmonary disease; $\mathrm{Cl}$, confidence interval; GOLD, Global initiative for chronic Obstructive Lung Disease; CKD, chronic kidney disease; eGFR, estimated glomerular filtration rate; ACEi, angiotensin-converting enzyme inhibitors; ARB, angiotensin receptor blocker.

drugs were all associated with lower mortality before and after adjusting for age, sex, and eGFR. Angina and antiplatelet medications were associated with lower mortality after adjustment for age and sex (Table 3).

AKI at the time of COPD exacerbation was associated with a substantially higher mortality rate (logrank: $P<0.001$ ), particularly during the first 6 months (Figure 2). In all, 50\% of patients without AKI survived approximately 5.5 years, while $54 \%$ of patients suffering from AKI survived 6 months. Mortality among patients with AKI at the first COPD exacerbation was $512 / 10^{3}$ py. The presence of AKI at the first COPD exacerbation during follow-up was associated with a 2.47-fold higher mortality rate after adjusting for age, sex, and eGFR (95\% CI: 2.24, 3.73) than those without AKI (Table 3). In the first 6 months after a hospitalization for COPD exacerbation, the risk of death was 1.80 (95\% CI: 1.61, 2.03) times higher in patients with concomitant AKI than those without after adjusting for age, sex, and baseline renal function, which reduced to 1.37 (95\% CI: $1.16,1.62)$ thereafter (Table 4$)$.

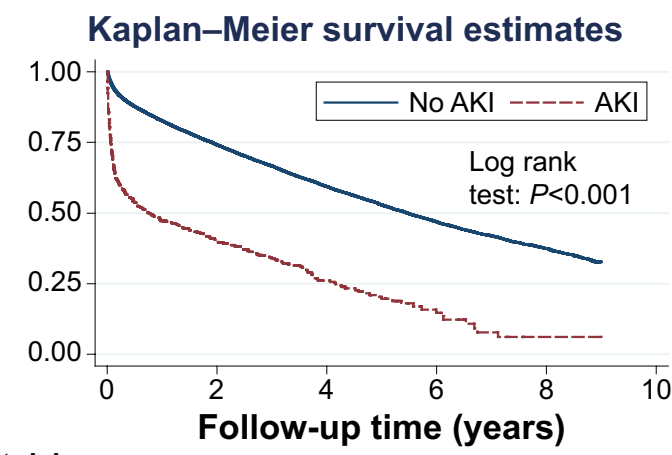

Number at risk

$\begin{array}{cllllll}\mathrm{AKI}=\text { no AKI } & 35,403 & 19,916 & 9,911 & 4,180 & 983 & 0 \\ \mathrm{AKI}=\mathrm{AKI} & 704 & 185 & 58 & 12 & 1 & 0\end{array}$

Figure 2 Kaplan-Meier survival curve stratified by AKI among patients with a COPD exacerbation $(n=36,107)$.

Abbreviations: AKI, acute kidney injury; COPD, chronic obstructive pulmonary disease.

\section{Discussion}

AKI is common among COPD patients who exacerbate. These people have a high mortality, particularly in the first 6 months after the event. This mortality is partially explained by having other comorbidities (eg, DM, HF, preexisting renal disease). However, the underlying comorbidities do not fully explain AKI mortality associations seen. This suggests that there are potentially modifiable factors that could prevent mortality associated with AKI in patients with COPD exacerbations.

The incidence rate of AKI admission in a COPD cohort was calculated to be $128 / 10^{5}$ py. The true incidence of AKI in the general population is not known, due to scarcity of studies. ${ }^{27}$ The strongest predictor of AKI is baseline renal function. This is supported by previous literature that

Table 4 Multivariable model of mortality following COPD exacerbation $(n=36,107)$

\begin{tabular}{|c|c|c|}
\hline Variable & Adjusted RR (95\% Cl) & $P$-value \\
\hline \multicolumn{3}{|c|}{$<6$ months follow-up } \\
\hline AKI & $1.80(1.61,2.03)$ & $<0.001$ \\
\hline \multicolumn{3}{|c|}{$\geq 6$ months follow-up } \\
\hline AKI & $1.37(1.16,1.62)$ & $<0.001$ \\
\hline \multicolumn{3}{|c|}{ Age at entry in years $(\operatorname{Ref}<50)$} \\
\hline$\geq 50,<60$ & $1.67(1.41,1.98)$ & $<0.001$ \\
\hline$\geq 60,<70$ & $2.83(2.41,3.34)$ & \\
\hline$\geq 70,<80$ & $4.06(3.45,4.78)$ & \\
\hline$\geq 80,<90$ & $5.4 \mathrm{I}(4.58,6.38)$ & \\
\hline$\geq 90$ & $6.90(5.70,8.35)$ & \\
\hline Sex: female & $0.89(0.86,0.92)$ & $<0.001$ \\
\hline \multicolumn{3}{|c|}{ eGFR in $\mathrm{mL} / \mathrm{kg} / \mathrm{l} .73 \mathrm{~m}^{2}(\operatorname{Ref} \geq 90)$} \\
\hline$\geq 60$ and $<90$ & $0.78(0.74,0.83)$ & $<0.001$ \\
\hline$\geq 30$ and $<60$ & $0.88(0.82,0.93)$ & \\
\hline$\geq 15$ and $<30$ & $1.04(0.95,1.13)$ & \\
\hline$<15$ & $1.02(0.84,1.24)$ & \\
\hline
\end{tabular}

Abbreviations: COPD, chronic obstructive pulmonary disease; RR, risk ratio; $\mathrm{Cl}$, confidence interval; $\mathrm{AKI}$, acute kidney injury; Ref, reference; eGFR, estimated glomerular filtration rate. 
suggests that CKD is a risk factor for AKI. ${ }^{11,12}$ Our study observed a similar trend in COPD patients. Patients likely to suffer from renal impairment (including microalbuminuria and proteinuria) had a larger observed incidence of AKI than those with known CKD, possibly due to the absence of education or treatment in this group. Being female is shown to be protective; AKI was $35 \%$ lower in females within this study sample. The risk of AKI increased with age and BMI. Other strong predictors (adjusted $\mathrm{RR} \geq 2.5$ ) included DM, $\mathrm{HF}$, and the use of diuretics and diabetic medications.

COPD severity does not have a rising effect on AKI. The incidence rate rises gradually but then drops at GOLD stage IV. This could be a result of the higher mortality rate in the most severe COPD group, meaning that they are more likely to die before achieving the outcome of interest (AKI hospitalization). ${ }^{28}$ The prevalence of concurrent AKI in COPD exacerbations is observed to be $1.9 \%$. There are no previous studies investigating the prevalence of AKI in COPD exacerbations. The predictors of concomitant AKI in COPD exacerbations are less abundant than those for AKI overall among the stable COPD cohort. Again, being female is observed to reduce the chance of the patient also having AKI by approximately one-third. Notably, age is negligible once eGFR and sex are taken into account.

The mortality rate of patients suffering from the first COPD exacerbation in the presence of concomitant AKI is $512 / 10^{3}$ py $(51.2 \%)$ compared to $138 / 10^{3}$ py $(13.8 \%)$ in those without AKI, producing a mortality RR of 3.71 (95\% CI: $3.39,4.07)$. According to the UK Renal Association, AKI is commonly seen in hospitalized patients with a mortality rate ranging from $10 \%$ to $80 \%$, which is consistent with this study's results. ${ }^{29}$ Uncomplicated AKI has a mortality rate of up to $10 \%$, while that with multiorgan failure holds a mortality rate of more than $50 \% .^{29}$ The estimated mortality rate of $>10 \%$ in this study shows consistency with previous studies, but comparison cannot be achieved with mortality associated with multiorgan failure as the extent of organ failure of the patients in this study is not known. A multicenter prospective study in the UK measured the COPD exacerbation mortality rate to be $23 \%$. ${ }^{30}$ Again, a $>23 \%$ mortality in COPD exacerbation in the presence of $\mathrm{AKI}$ is consistent with previous literature.

The mortality rate, adjusting for age and eGFR, was estimated to be $13 \%$ lower in females compared to males. As expected, mortality increases with older age groups despite sex and baseline renal function. Higher than normal BMIs are protective in the context of COPD exacerbations adjusting for age, sex, and renal function, while the opposite is observed for lower BMIs. This trend is consistent with the BODE index, which suggests that patients with $\mathrm{BMI} \geq 21 \mathrm{~kg} / \mathrm{m}^{2}$ are at a significantly lower risk of death than those $<21 \mathrm{~kg} / \mathrm{m}^{2} .{ }^{31}$ Mortality expectedly increases with worsening COPD (according to GOLD staging), which is compatible with Mannino et al's findings. ${ }^{28}$ Other than baseline renal function and degree of COPD, we did not find any other strong predictors.

Similar to previous studies, we demonstrated higher mortality rates closer to the time of COPD exacerbation. ${ }^{32,33}$ There is a notable steep increase in deaths within the first 6 months post COPD exacerbation, contributing to a $>50 \%$ mortality in the first year after COPD exacerbation in patients with concomitant AKI.

\section{Limitations}

AKI is a known adverse effect of nonsteroidal anti-inflammatory drugs. Nonsteroidal anti-inflammatory drugs are available over the counter without a prescription, making recording these data almost impossible. This study relies on GP documentation of death (from CPRD), and the date of death recorded may not be accurate. Of the total 189,561 COPD patients, 125,364 (66\%) were lost to follow-up over the 9 years.

We calculated eGFR without adjusting for ethnicity. Patients of Black ethnicity produce higher eGFR on estimation, and therefore, some patients' baseline renal functions are underestimated causing differential misclassification of eGFR. However, according to the 2011 Census, the Black population only comprises $3 \%$ of the UK population; hence, this issue is likely to have a negligible effect on the associations seen. ${ }^{34}$

Information provided on CKD status is potentially biased as patients at high risk of kidney disease (such as diabetics and hypertensive patients) are monitored closer for signs of CKD than those with no risk factors. Additionally, assuming that those without CKD codes have no kidney disease is probably conservative, as we are likely to have missed more cases. Findings are indicative of underrecognition of kidney problems in people with COPD as evidenced by finding a subgroup of people with severe kidney function impairments without corresponding primary care CKD Read Code.

Patients required a COPD diagnosis for inclusion: some patients with stable COPD and less severe breathing difficulty may not seek any medical attention. This raises the issue of selection bias, as the COPD patients selected for the study are not a true representative of the population distribution of COPD severity. Furthermore, on identifying patients hospitalized with COPD exacerbation, ICD-10 codes are used. These codes may be presented inaccurately. There is a certain ICD-10 code for patients admitted into hospital with 
COPD exacerbation; separate codes for lower respiratory tract infection and pneumonia are also available to choose from. COPD patients coded for a chest infection may have also had an exacerbation, and therefore, the prevalence of COPD exacerbation among COPD patients is underestimated. Moreover, the COPD exacerbation sample is not representative of the true COPD exacerbations. The same can be said about the AKI codes. Since 2008, the rates of AKI coding have been steadily increasing (note that this cohort study started in 2004), hence again, underestimation of clinical condition. ${ }^{35}$

Potential confounding factors are assessed during this study; however, unavailable and unknown potential confounders are not considered. Most notably, smoking status and socioeconomic status could confound the association between AKI and mortality.

This study had prespecified investigation of mortality in COPD exacerbations and concomitant AKI versus COPD exacerbation alone. Since we are already aware that having AKI increases mortality, it would also be interesting to see if patients with concurrent AKI and COPD exacerbations have a higher mortality rate than AKI alone. ${ }^{36}$ Additionally, we did not have information on different causes of AKI in this population. It would be interesting to assess whether COPD patients have more prerenal AKI related to comorbidities or more postrenal AKI due to anticholinergic use for example.

In conclusion, AKI is considered a predictor of poor outcome in patients with COPD exacerbation. Consequently, it is recommended for patients suffering from simultaneous AKI and COPD exacerbation to be identified as higher risk patients and hence monitored more closely, especially early after hospital discharge.

\section{Acknowledgments}

Dr Quint reports grants from Medical Research Council, during the conduct of the study, and grants from Medical Research Council, grants and personal fees from GSK, grants from British Lung Foundation, and travel support from AstraZeneca, outside the submitted work.

\section{Disclosure}

The authors report no conflicts of interest in this work.

\section{References}

1. World Health Organization. Chronic Obstructive Pulmonary Disease (COPD); 2014 [cited July 23, 2014]; Available from: http://www.who. int/respiratory/copd/en/

2. Halbert RJ, Natoli JL, Gano A, Badamgarav E, Buist AS, Mannino DM. Global burden of COPD: systematic review and meta-analysis. Eur Respir J. 2006;28(3):523-532.
3. NHS. A Strategic Approach to Prevention and Early Identification of COPD. NHS Improvement 2011; 2011. Available from: http://www. nhsiq.nhs.uk/legacy-websites/nhs-improvement.aspx

4. Fabbri LM, Luppi F, Beghé B, Rabe KF. Complex chronic comorbidities of COPD. Eur Respir J. 2008;31(1):204-212.

5. Corsonello A, Antonelli Incalzi R, Pistelli R, Pedone C, Bustacchini S, Lattanzio F. Comorbidities of chronic obstructive pulmonary disease. Curr Opin Pulm Med. 2011;17(suppl 1):S21-S28.

6. Global Initiative for Chronic Obstructive Lung Disease. Global Strategy for Diagnosis, Management and Prevention of COPD; 2010. [cited July 25, 2014]. Available from: www.goldcopd.org

7. Gjerde B, Bakke PS, Ueland T, Hardie JA, Eagan TM. The prevalence of undiagnosed renal failure in a cohort of COPD patients in western Norway. Respir Med. 2012;106(3):361-366.

8. Incalzi RA, Corsonello A, Pedone C, et al; Extrapulmonary Consequences of COPD in the Elderly Study Investigators. Chronic renal failure: a neglected comorbidity of COPD. Chest. 2010;137(4): 831-837.

9. Stevens PE, Tamimi NA, Al-Hasani MK, et al. Non-specialist management of acute renal failure. QJM. 2001;94(10):533-540.

10. National Institute of Clinical Excellence. Acute Kidney Injury: Prevention, Detection and Management of Acute Kidney Injury Up To The Point of Renal Replacement Therapy; 2013 [cited July 8, 2015]. Available from: http://www.nice.org.uk/guidance/cg169/documents/ acute-kidney-injury-nice-version2

11. Hsu CY, Ordoñez JD, Chertow GM, Fan D, McCulloch CE, Go AS. The risk of acute renal failure in patients with chronic kidney disease. Kidney Int. 2008;74(1):101-107.

12. Singh P, Rifkin DE, Blantz RC. Chronic kidney disease: an inherent risk factor for acute kidney injury? Clin J Am Soc Nephrol. 2010;5(9): 1690-1695.

13. James MT, Hemmelgarn BR, Wiebe N, et al; Alberta Kidney Disease Network. Glomerular filtration rate, proteinuria, and the incidence and consequences of acute kidney injury: a cohort study. Lancet. 2010; 376(9758):2096-2103.

14. Lameire N, Van Biesen W, Vanholder R. Acute renal failure. Lancet. 2005;365(9457):417-430.

15. Wedzicha JA, Seemungal TA. COPD exacerbations: defining their cause and prevention. Lancet. 2007;370(9589):786-796.

16. Sharkey RA, Mulloy EM, O'Neill SJ. The acute effects of oxygen and carbon dioxide on renal vascular resistance in patients with an acute exacerbation of COPD. Chest. 1999;115(6):1588-1592.

17. Sofia M, Maniscalco M, Celentano L, et al. Abnormalities of renal endothelin during acute exacerbation in chronic obstructive pulmonary disease. Pulm Pharmacol Ther. 2001;14(4):321-327.

18. Palange $P$. Renal and hormonal abnormalities in chronic obstructive pulmonary disease (COPD). Thorax. 1998;53(11):989-991.

19. Sloane J, Wilson J, Griffin C, Wilkie M, Chalmers J, Schembri S., Elevated creatinine is a sensitive severity marker in community acquired pneumonia. In: European Respiratory Society Annual Congress, Amsterdam; 2012.

20. Clinical Practice Research Datalink. About us (CPRD); 2014 [cited July 30, 2014]. Available from: http://www.cprd.com/about/

21. Health and Social Care Information Centre. Hospial Episode Statistics; 2014 [cited July 30, 2014]. Available from: http://www.hscic.gov.uk/hes

22. Quint JK, Müllerova H, DiSantostefano RL, et al. Validation of chronic obstructive pulmonary disease recording in the Clinical Practice Research Datalink (CPRD-GOLD). BMJ Open. 2014;4(7):1-8.

23. Pauwels RA, Buist AS, Ma P, Jenkins CR, Hurd SS; GOLD Scientific Committee. Global strategy for the diagnosis, management, and prevention of chronic obstructive pulmonary disease: National Heart, Lung, and Blood Institute and World Health Organization Global Initiative for Chronic Obstructive Lung Disease (GOLD): executive summary. Respir Care. 2001;46(8):798-825.

24. World Health Organization. BMI Classification; 2006 [cited August 5, 2013]. Available from: http://apps.who.int/bmi/index. jsp?introPage=intro_3.html. Accessed August 15, 2015. 
25. Levey AS, Stevens LA, Schmid CH, et al; CKD-EPI (Chronic Kidney Disease Epidemiology Collaboration). A new equation to estimate glomerular filtration rate. Ann Intern Med. 2009;150(9):604-612.

26. Matsushita K, Mahmoodi BK, Woodward M, et al; Chronic Kidney Disease Prognosis Consortium. Comparison of risk prediction using the CKD-EPI equation and the MDRD study equation for estimated glomerular filtration rate. JAMA. 2012;307(18):194151.

27. Cerda J, Lameire N, Eggers P, et al. Epidemiology of acute kidney injury. Clin J Am Soc Nephrol. 2008;3(3):881-886.

28. Mannino DM, Doherty DE, Sonia Buist A. Global Initiative on Obstructive Lung Disease (GOLD) classification of lung disease and mortality: findings from the atherosclerosis risk in communities (ARIC) study. Respir Med. 2006;100(1):115-122.

29. Lewington A, Kanagasundaram S. Acute Kidney Injury. Hampshire: UK Renal Association; 2011.

30. Seemungal TA, Hurst JR, Wedzicha JA. Exacerbation rate, health status and mortality in COPD - a review of potential interventions. Int J Chron Obstruct Pulmon Dis. 2009;4:203-223.
31. Celli BR. Predictors of mortality in COPD. Respir Med. 2010;104(6): 773-779.

32. Chung LP, Winship P, Phung S, Lake F, Waterer G. Five-year outcome in COPD patients after their first episode of acute exacerbation treated with non-invasive ventilation. Respirology. 2010;15(7):1084-1091.

33. Marin JM, Alfageme I, Almagro P, et al. Multicomponent indices to predict survival in COPD: the COCOMICS study. Eur Respir J. 2013; 42(2):323-332.

34. Office of National Statistics. 2011 Census; 2011 [cited July 23, 2014]. Available from: http://www.ons.gov.uk/ons/rel/census/2011-census/

35. Kolhe N. Epidemiology of Acute Kidney Injury in England - 1998 to 2013; 2014 [cited April 29, 2015]. Available from: https://clinicaltrials. gov/ct2/show/NCT02216695

36. Chertow GM. Acute kidney injury, mortality, length of stay, and costs in hospitalized patients. J Am Soc Nephrol. 2005;16:3365-3370.
International Journal of COPD

\section{Publish your work in this journal}

The International Journal of COPD is an international, peer-reviewed journal of therapeutics and pharmacology focusing on concise rapid reporting of clinical studies and reviews in COPD. Special focus is given to the pathophysiological processes underlying the disease, intervention programs, patient focused education, and self management protocols.

\section{Dovepress}

This journal is indexed on PubMed Central, MedLine and CAS. The manuscript management system is completely online and includes a very quick and fair peer-review system, which is all easy to use. Visit http://www.dovepress.com/testimonials.php to read real quotes from published authors.

Submit your manuscript here: http://www.dovepress.com/international-journal-of-chronic-obstructive-pulmonary-disease-journal 